\title{
RÉNYI ENTROPIES OF APERIODIC DYNAMICAL SYSTEMS
}

\author{
FLORIS TAKENS AND EVGENY VERBITSKIY
}

\begin{abstract}
In this paper we continue the study of Rényi entropies of measurepreserving transformations started in [22]. We have established there that for ergodic transformations with positive entropy, the Rényi entropies of order $q, q \in \mathbb{R}$, are equal to either plus infinity $(q<1)$, or, to the measure-theoretic (KolmogorovSinai) entropy $(q \geq 1)$. The answer for non-ergodic transformations is different: the Rényi entropies of order $q>1$ are equal to the essential infimum of the measuretheoretic entropies of measures forming the decomposition into ergodic components. Thus, it is possible that the Rényi entropies of order $q>1$ are strictly smaller than the measure-theoretic entropy, which is the average value of entropies of ergodic components.

This result is a bit surprising: the Rényi entropies are metric invariants, which are sensitive to ergodicity.

The proof of the described result is based on the construction of partitions with independent iterates. However, these partitions are obtained in different ways depending on $q$ : for $q>1$ we use a version of the well-known Sinai theorem on Bernoulli factors for the non-ergodic transformations; for $q<1$ we use the notion of collections of independent sets in Rokhlin-Halmos towers and their properties.
\end{abstract}

\section{INTRODUCTION}

Alfred Rényi introduced the generalization of the Shannon information (entropy) in the beginning of sixties. His approach was based on an axiomatic definition of information, and consisted of including the standard entropy function

$$
H\left(p_{1}, \ldots, p_{n}\right)=-\sum_{i=1}^{n} p_{i} \log p_{i},
$$

into a one-parameter family of generalized entropy functions

$$
H_{q}\left(p_{1}, \ldots, p_{n}\right)=-\frac{1}{q-1} \log \left(\sum_{i=1}^{n} p_{i}^{q}\right), \quad q \neq 1 .
$$

For a fixed probability distribution $\left(p_{1}, \ldots, p_{n}\right)$ the standard entropy is recovered from the generalized entropies as follows

$$
H\left(p_{1}, \ldots, p_{n}\right)=\lim _{q \rightarrow 1} H_{q}\left(p_{1}, \ldots, p_{n}\right)
$$

Since then the Rényi entropies have been successfully used in information theory and statistics, and more recently in thermodynamics and quantum mechanics. In dynamical systems, Hentschel and Procaccia [8] suggested a one-parameter family of generalized dimensions based on Rényi's approach. These dimensions proved to be

Date: December 21, 2000. 
extremely useful in problems of multifractal analysis and characterization of chaotic attractors, see e.g. [13].

Some attempts [7], [6] were made to introduce the generalized entropies of dynamical system using Rényi's approach. The idea was to produce a sufficiently rich family of invariants of a dynamical system, which will take into account the non-uniform behavior of invariant measures. However, the proposed way of generalizing the Kolmogorov-Sinai entropy using $H_{q}$ instead of $H_{1}$, turned out to be non-productive. In [22] we have established the following fact.

Theorem 1.1. For an ergodic dynamical system $(X, \mathfrak{B}, \mu, T)$ with positive measuretheoretic entropy $h(T, \mu)>0$, the Rényi entropies are given by the following formula

$$
h(T, q, \mu)= \begin{cases}+\infty, & q<1 \\ h(T, \mu), & q \geq 1 .\end{cases}
$$

Also in [22] we suggested another family of generalized entropies, which recovers the results reported in the physics literature [1].

The proof of Theorem 1.1 relies heavily on Sinai's theorem on Bernoulli factors [19], for which the assumptions of ergodicity and positiveness of the measure-theoretic entropy are crucial.

In this paper we prove a result, similar to Theorem 1.1, but without the above assumptions. We consider aperiodic measure-preserving automorphisms, i.e., transformations $T$ of some Lebesgue space $(X, \mathfrak{B}, \mu)$ such that

$$
\mu\left(\left\{x: T^{n}(x)=x \text { for some } n\right\}\right)=0 .
$$

Surprisingly, the result for such systems is different from the ergodic case.

Theorem 1.2. Suppose $T$ is an aperiodic measure-preserving automorphism of the Lebesgue space $(X, \mathfrak{B}, \mu)$. Let $\mu=\int \mu_{t} d m(t)$ be the decomposition of $\mu$ into ergodic components, and let

$$
h_{*}(T, \mu)=\mathrm{m}-\operatorname{essinf}\left\{h\left(T, \mu_{t}\right)\right\}:=\sup \left\{c: m\left\{t: h\left(T, \mu_{t}\right)<c\right\}=0\right\}
$$

Then the Rényi entropies are as follows

$$
h(T, q, \mu)= \begin{cases}+\infty, & q<1, \\ h(T, \mu)=\int h\left(T, \mu_{t}\right) d m(t), & q=1 \\ h_{*}(T, \mu), & q>1 .\end{cases}
$$

This result is a bit surprising because of the following: an entropy-based invariant can detect ergodicity. However, we are not aware of any interesting example, where this observation could be useful. The first candidates, which come to mind, are the non-ergodic Markov shifts, i.e., the shifts for which the transition probability matrix $P$ is not irreducible. It is possible in this case (provided $h(T, \mu)>h_{*}(T, \mu)$, of course) to show the Rényi entropies of order $q>1$ are strictly smaller than the measuretheoretic entropy, and thus, the system is not ergodic. However, this proof is much more involved than the standard one, and follows the same idea.

The paper is organized as follows: in the next section we give a formal definition of the Rényi entropies and establish the basic properties; in section 3 we recall facts about the decomposition into ergodic components. We discuss a non-ergodic version 
of Sinai's theorem on Bernoulli factors, and use it for the computation of the Rényi entropies of order $q>1$ in section 4 . In section 5 we develop a notion of independent partitions in Rokhlin-Halmos towers and subsequently prove the statement for $q<1$. Finally, in the last section, we pose some open questions about the possible connection between the Rényi entropiesand the recently introduced entropy convergence rates.

\section{RÉNYi ENTROPIES OF MEASURE PRESERVING TRANSFORMATIONS}

The definition of the Rényi entropy of order $q$ of a measure-preserving transformation goes along the lines of the standard definition of the measure-theoretic (Kolmogorov-Sinai) entropy, and consists of 3 steps: the definition of the Rényi entropy of a finite partition, Rényi entropy of an automorphism with respect to a partition, and, finally, after taking the supremum over all finite partitions, the Rényi entropy of an automorphism, which is a metric invariant.

For any $q \in \mathbb{R}$ the entropy of order $q$ of the partition $\xi=\left\{\Delta_{i}\right\}_{i=1}^{n}$ is the number

$$
H_{\mu}(q, \xi)= \begin{cases}-\frac{1}{q-1} \log \left(\sum_{i=1}^{n} \mu\left(\Delta_{i}\right)^{q}\right), & \text { for } q \neq 1, \\ -\sum_{i=1}^{n} \mu\left(\Delta_{i}\right) \log \mu\left(\Delta_{i}\right), & \text { for } q=1,\end{cases}
$$

with the standard convention $0^{q}=0$ for all $q \in \mathbb{R}$ and $0 \log 0=0$.

It is easy to check the following monotonicity property

$$
H_{\mu}\left(q_{1}, \xi\right) \leq H_{\mu}\left(q_{2}, \xi\right) \text { for any } \xi \text { and } q_{1} \geq q_{2} .
$$

The Rényi entropy of order $q$ with respect to a partition $\xi$ is defined as

$$
h(T, \mu, q, \xi)=\liminf _{n \rightarrow \infty} \frac{1}{n} H_{\mu}\left(q, \xi^{(n)}\right),
$$

where $\xi^{(n)}=\xi \vee T^{-1} \xi \vee \ldots \vee T^{-n+1} \xi$ is the partition into sets $\bigcap_{k=0}^{n-1} T^{-k} \Delta_{i_{k}}$ with $\Delta_{i_{k}} \in \xi$.

Remark. For $q=1$ it is known (see for example [4]) that the limit in (2.2) exists. The proof of this fact is based on a so-called subadditivity property of the Shannon entropy $H(1, \xi)$ :

$$
H_{\mu}(1, \xi \vee \eta) \leq H_{\mu}(1, \xi)+H_{\mu}(1, \eta)
$$

for all partitions $\xi, \eta$. As it was shown by Rényi in [14], the later is not the case for any $q \neq 1$. This creates some additional problems in the treatment of the Rényi entropies. Nevertheless, if $\xi$ and $\eta$ are independent partitions then

$$
H_{\mu}(q, \xi \vee \eta)=H_{\mu}(q, \xi)+H_{\mu}(q, \eta)
$$

for all $q \in \mathbb{R}$. We will often exploit this fact.

Finally, we define the Rényi entropy of an automorphism $T$ of order $q$ as the number

$$
h(T, \mu, q)=\sup _{\xi} h(T, \mu, q, \xi),
$$

where the supremum is taken over all finite partitions $\xi$ of $X$.

Proposition 2.1. The Rényi entropies have the following properties:

1) $h(T, \mu, q) \geq 0$ for all $q$; 
2) $h\left(T, \mu, q_{1}\right) \geq h\left(T, \mu, q_{2}\right)$ for $q_{1} \leq q_{2}$;

3) $h(T, \mu, 1)=h(T, \mu)$, where $h(T, \mu)$ is the measure-theoretic (or KolmogorovSinai) entropy.

4) $h\left(T^{n}, \mu, q\right)=n h(T, \mu, q)$ for any $q \in \mathbb{R}$ and every $n \geq 0$.

Properties 1-3 follow easily from the definition of $h(T, \mu, q)$, and 4 has been established in [22].

\section{Decomposition into ERgodic COMponents}

Let $(X, \mathfrak{B}, \mu)$ be a Lebesgue space [4]. For a measurable partition $\xi=\left\{C_{t}\right\}_{t \in \Lambda}$, where $\Lambda$ can be finite, countable or uncountable, we identify $\Lambda$ and the quotient (or, factor) $X / \xi$ - the space, whose points are the elements of $\xi$. The set $\Lambda$ is a Lebesgue space as well: the set $E \subset \Lambda$ is measurable if the set $\cup_{t \in E} C_{t}$ is a measurable subset of $X$, and we obtain a measure $m$ on $\Lambda$ by letting $m(E)=\mu\left(\cup_{t \in E} C_{t}\right)$. A system of measures $\left\{\mu_{t}\right\}, t \in \Lambda$, is called a canonical system of conditional measures belonging to the partition $\xi=\left\{C_{t}\right\}_{t \in \Lambda}$, if

1) $\mu_{t}$ is defined on some $\sigma$-algebra $\mathfrak{B}_{t}$ of subsets of $C_{t}$, such that $\left(C_{t}, \mathfrak{B}_{t}, \mu_{t}\right)$ is a Lebesgue space.

2) for any $A \in \mathfrak{B}$ the set $A \cap C_{t}$ belongs to $\mathfrak{B}_{t}$ for $m$-almost all $t$; the function $\mu_{t}\left(A \cap C_{t}\right)$ is a measurable function of $t$ and

$$
\mu(A)=\int \mu_{t}\left(A \cap C_{t}\right) d m(t)
$$

Suppose $T: X \rightarrow X$ is a measure-preserving automorphism. Then $(X, \mathfrak{B}, \mu)$ can be decomposed into ergodic components of $T$. By this we mean the following: there exists a $T$-invariant measurable partition $\xi=\left\{C_{t}\right\}$ and a canonical system of conditional measures $\left\{\mu_{t}\right\}$ such that for almost all $t$

$$
\left(C_{t}, \mathfrak{B}_{t}, \mu_{t},\left.T\right|_{C_{t}}\right) \quad \text { is ergodic. }
$$

Suppose $\xi=\left\{C_{t}\right\}$ is the decomposition into ergodic components of $(X, \mathfrak{B}, \mu, T)$, then

$$
h(T, \mu)=\int h\left(T, \mu_{t}\right) d m .
$$

Consider the essential infimum and the essential supremum of measure-theoretic entropies of the measures $\mu_{t}$ from the decomposition into ergodic components:

$$
\begin{aligned}
& h_{*}(T, \mu)=\mathrm{m}-\operatorname{essinf}\left\{h\left(T, \mu_{t}\right) \mid t \in \Lambda=X / \xi\right\}:=\sup \left\{c: m\left(\left\{t: h\left(T, \mu_{t}\right)<c\right\}\right)=0\right\}, \\
& h^{*}(T, \mu)=\mathrm{m}-\operatorname{esssup}\left\{h\left(T, \mu_{t}\right) \mid t \in \Lambda=X / \xi\right\}:=\inf \left\{c: m\left(\left\{t: h\left(T, \mu_{t}\right)>c\right\}\right)=0\right\} .
\end{aligned}
$$

The quantity $h^{*}(T, \mu)$, sometimes called the entropy rate, has been previously studied in the literature $[9,21,23]$ in relation with the existence of finite generators (generating partitions) for non-ergodic systems. A well-known theorem of Krieger [11] states that an ergodic dynamical system with a finite measure-theoretic entropy $h(T, \mu)$ admits a finite generator $\xi$ with $\operatorname{card}(\xi) \leq \exp (h(T, \mu))+1$. It turns out that for nonergodic aperiodic dynamical systems a similar result is true, provided $h^{*}(T, \mu)<\infty$ : a finite generator $\xi$ exists whose cardinality does not exceed $\exp \left(h^{*}(T, \mu)\right)+1$. 
Denote by $\Pi_{m}=\left\{P=\left(P_{1}, \ldots, P_{m}\right)\right\}$ the set of all ordered partitions of $X$ into $m$ sets. For any measure $\mu$ on $(X, \mathfrak{B})$ define the partition (pseudo-) metric $\rho_{\mu}$ on $\Pi_{m}$ as follows

$$
\rho_{\mu}(P, Q)=\sum_{k=1}^{m} \mu\left(P_{k} \triangle Q_{k}\right), \quad P, Q \in \Pi_{m} .
$$

If $\rho_{\mu}(P, Q)=0$ then $P$ and $Q$ agree except on a set of measure 0 , and, of course, in this case we say that $P=Q$. The space $\left(\Pi_{m}, \rho_{\mu}\right)$ is a complete metric space.

For an at most countable ordered partition $P$ of $(X, \mathfrak{B}, \mu)$ the distribution vector of $P$ is given by

$$
d(P, \mu)=\left(\mu\left(P_{1}\right), \mu\left(P_{2}\right), \ldots\right) .
$$

Suppose $P$ and $\bar{P}$ are partitions into $m$ sets of $(X, \mathfrak{B}, \mu),(Y, \mathcal{F}, \nu)$ respectively, then the distribution distance is

$$
|d(P, \mu)-d(\bar{P}, \nu)|:=\sum_{k=1}^{m}\left|\mu\left(P_{k}\right)-\nu\left(\bar{P}_{k}\right)\right| .
$$

Suppose we have a set $\left\{\mu_{t}\right\}_{t \in \Lambda}$ of measures on $(X, \mathfrak{B})$. For every $t \in \Lambda$ consider the metric $\rho_{\mu_{t}}$ on $\Pi_{m}$. The following fact will be used later: there exists a countable set $\tilde{\Pi}_{m} \subset \Pi_{m}$, which is $\rho_{\mu_{t}}$-dense in $\Pi$ for almost every $t \in \Lambda$.

The existence of such $\tilde{\Pi}_{m}$ follows from the fundamental properties of the Lebesgue spaces. By definition, a Lebesgue space $(X, \mathfrak{B}, \mu)$ admits a countable basis $\Gamma=\left\{B_{\alpha}\right\}$. This in particular means that for any measurable set $A \in \mathfrak{B}$ there exists a set $C$ from a minimal $\sigma$-algebra generated by $\Gamma$ such that

$$
C \subset A \text { and } \mu(A \backslash C)=0 .
$$

Denote by $\mathfrak{A}$ the countable algebra generated by $\Gamma$, and let

$$
\tilde{\Pi}_{m}=\left\{P=\left(P_{1}, \ldots, P_{m}\right): P_{i} \in \mathfrak{A}\right\} .
$$

Hence $\tilde{\Pi}_{m}$ is an at most countable collection of ordered partitions into $m$ sets, where elements of these partitions are taken from $\mathfrak{A}$. From (3.1) we conclude that $\tilde{\Pi}_{m}$ is $\rho_{\mu}$ dense in $\Pi_{m}$. Moreover, for almost every $t \in \Lambda, \tilde{\Pi}_{m}$ is $\rho_{\mu_{t}}$-dense in $\Pi_{m}$ as well. This is a consequence of the following fact ([15], see also [16]): for almost every $t \in \Lambda$, the countable collection of sets $\Gamma_{t}=\Gamma \cap C_{t}$ is a basis in the Lebesgue space $\left(C_{t}, \mathfrak{B}_{t}, \mu_{t}\right)$.

\section{RÉNYI ENTROPIES OF ORDER $q>1$}

In this section we are going to prove that $h(T, \mu, q)=h_{*}(T, \mu)$ for every $q>1$. We start by showing that $h(T, \mu, q) \leq h_{*}(T, \mu)$.

4.1. Estimate from above. Suppose that we have two invariant measures $\mu_{1}$ and $\mu_{2}$ for an automorphism $T$. We do not assume these measures to be ergodic. Without loss of generality we can assume that

$$
h\left(T, \mu_{1}\right) \leq h\left(T, \mu_{2}\right) .
$$

Consider now another invariant measure $\mu=\alpha \mu_{1}+(1-\alpha) \mu_{2}$ with $\alpha \in(0,1)$. The measure-theoretic entropy of $\mu$ is given by (see [5])

$$
h(T, \mu)=\alpha h\left(T, \mu_{1}\right)+(1-\alpha) h\left(T, \mu_{2}\right) .
$$


Note that due to $(4.1) h(T, \mu) \geq h\left(T, \mu_{1}\right)$. Let $\xi$ be some finite partition. For any $C \in \xi$ one has

$$
\mu(C)=\alpha \mu_{1}(C)+(1-\alpha) \mu_{2}(C),
$$

and, therefore, $\mu(C)^{q} \geq \alpha^{q} \mu_{1}(C)^{q}$ for $q>1$. Hence, for $q>1$,

$$
\begin{aligned}
H_{\mu}(q, \xi)=-\frac{1}{q-1} \log \left(\sum_{C \in \xi} \mu(C)^{q}\right) & \leq-\frac{q}{q-1} \log \alpha-\frac{1}{q-1} \log \left(\sum_{C \in \xi} \mu_{1}(C)^{q}\right) \\
& =-\frac{q}{q-1} \log \alpha+H_{\mu_{1}}(q, \xi) .
\end{aligned}
$$

From the above one easily concludes that

$$
h(T, \mu, q, \xi)=\liminf _{n \rightarrow \infty} \frac{1}{n} H_{\mu}\left(q, \xi^{(n)}\right) \leq \liminf _{n \rightarrow \infty} \frac{1}{n} H_{\mu_{1}}\left(q, \xi^{(n)}\right)=h\left(T, \mu_{1}, q, \xi\right) .
$$

On the other hand, due to the monotonicity of the Rényi entropies with respect to $q$, for $q>1$ we have

$$
h\left(T, \mu_{1}, q, \xi\right) \leq h\left(T, \mu_{1}, q\right) \leq h\left(T, \mu_{1}, 1\right)=h\left(T, \mu_{1}\right) .
$$

Combining the two last inequalities we finally obtain that for any $q>1$

$$
h(T, \mu, q) \leq h\left(T, \mu_{1}\right) .
$$

Thus we see that the Rényi entropy of a linear combination of two measures does not exceed a minimum of the measure-theoretic entropies of these two measures. It is evident that the above argument goes through in the case of a finite or countable decomposition: $\mu=\sum_{k} \alpha_{k} \mu_{k}$, where $\alpha_{k} \geq 0$ and $\sum_{k} \alpha_{k}=1$.

Moreover, the above argument can be equally easily generalized to the case of, generally, uncountable decomposition of an invariant measure $\mu$ into ergodic components $\left\{\mu_{t}\right\}$. This is done in the following lemma.

Lemma 4.1. For a measure preserving system $(X, \mathfrak{B}, \mu, T)$ one has

$$
h(T, \mu, q) \leq h_{*}(T, \mu)
$$

for every $q>1$.

Proof. Consider an ergodic decomposition of $(X, \mathfrak{B}, \mu, T)$ as in section 3. By the definition of $h_{*}(T, \mu)$ for every $\varepsilon>0$ the set $E_{1}=\left\{t: h\left(T, \mu_{t}\right)<h_{*}(T, \mu)+\varepsilon\right\}$ has a positive $m$-measure. Suppose, there exists $\varepsilon_{0}>0$ such that for any $\varepsilon \in\left(0, \varepsilon_{0}\right)$ one has $m\left(E_{1}\right)<1$.

If such $\varepsilon_{0}>0$ does not exist, then

$$
h\left(T, \mu_{t}\right)=h_{*}(T, \mu) \text { for } m \text {-a.a. } t .
$$

As a result we immediately conclude that $h(T, \mu)=h_{*}(T, \mu)$, and using the fact that $h(T, \mu) \geq h(T, \mu, q)$ for any $q>1$ we obtain our claim (4.2).

Assume such $\varepsilon_{0}>0$ exists and chose any $\varepsilon \in\left(0, \varepsilon_{0}\right)$. Since $m\left(E_{1}\right) \in(0,1)$ we can define

$$
\mu_{1}=\frac{1}{m\left(E_{1}\right)} \int_{E_{1}} \mu_{t} d m(t), \quad \mu_{2}=\frac{1}{1-m\left(E_{1}\right)} \int_{E_{1}^{c}} \mu_{t} d m(t) .
$$


It is clear that $\mu_{1}$ and $\mu_{2}$ are invariant probability measures. Moreover, $h\left(T, \mu_{1}\right) \leq$ $h_{*}(T, \mu)+\varepsilon$. Using the above argument for two measures $\mu_{1}$ and $\mu_{2}$ we conclude that for any $q>1$

$$
h(T, \mu, q) \leq h_{*}(T, \mu)+\varepsilon .
$$

Since $\varepsilon>0$ can be chosen arbitrary small, we obtain the claim (4.2).

4.2. Bernoulli factors of non-ergodic systems. Let us recall a definition of a Bernoulli automorphism.

Definition 4.2. An automorphism $T$ of a Lebesgue space $(X, \mathfrak{B}, \mu)$ is called Bernoulli, if it is measure-theoretically isomorphic to a Bernoulli shift.

If $T$ is a Bernoulli automorphism then there exists a partition $P$ of $X$ such that

1) $P$ is generating,

2) $\left\{T^{n} P\right\}_{n \in \mathbb{Z}}$ is a sequence of independent partitions.

Such partition $P$ is called an independent generator for $T$. A well known theorem by Sinai [19] states that for every ergodic automorphism $T$ with entropy $h(T, \mu)$, and every positive number $h$ such that $h \leq h(T, \mu)$, there exists a Bernoulli factor with entropy $h$. A non-ergodic version of the Sinai theorem fistt appeared in [10].

Theorem 4.3. Suppose $T$ is an automorphism of a Lebesgue space $(X, \mathfrak{B}, \mu)$. Let $\bar{T}$ be a Bernoulli automorphism of $(Y, \mathfrak{F}, \nu)$ with a finite independent generator $\bar{P}$, $\operatorname{card}(\bar{P})=k$. Let $\left\{C_{t}, \mu_{t}\right\}$ be a decomposition of $(X, \mathfrak{B}, \mu, T)$ into ergodic components and $m$ be a corresponding measure on the factor $X /\left\{C_{t}\right\}$. Assume that $h_{*}(T, \mu) \geq$ $h(\bar{T}, \nu)$. Then there exists a partition $Q, \operatorname{card}(Q)=k$, such that

i) $\left\{T^{i} Q\right\}$ is a sequence of independent partitions,

ii) $d(Q, \mu)=d(\bar{P}, \nu)$.

In fact, using the techniques of [10] one can establish a non-ergodic version of the Ornstein fundamental lemma $[12,18,20]$ as well. The strategy of generalizing "ergodic" results to the non-ergodic case consists of the following. Suppose that $\left\{C_{t}, \mu_{t}\right\}$ is the decomposion of $\mu$ into ergodic components, and that for almost every $t$ there exists a partition $P_{t}$ of $C_{t}$ into $m$ elements which satisfies some required property. We recall that there exists a countable family of partitions $\tilde{\Pi}_{m}$ which is $\rho_{\mu_{t}}$-dense in the set all partitions into $m$ elements for almost all $t$, see section 3 . Using this family $\tilde{\Pi}_{m}$ one can construct a universal partition $P$ such that $P \cap C_{t}=P_{t}$ for almost all $t$.

4.3. Estimate from below. Now we can prove a lower estimate: $h(T, \mu, q) \geq$ $h_{*}(T, \mu)$ for all $q>1$. Before we proceed with this estimate we would like to make a few remarks. Firstly, we compute the Rényi entropy of order $q$ for a Bernoulli shift. Let $\Omega=\{1, \ldots, k\}^{\mathbb{Z}}$, and $\sigma: \Omega \rightarrow \Omega$ be a left shift. Let $\nu$ be a Bernoulli measure on $\Omega$ generated by a probability vector $p=\left(p_{1}, \ldots, p_{k}\right)$, i.e.,

$$
\nu\left\{\omega=\left(\omega_{i}\right): \omega_{m}=a_{m}, \ldots, \omega_{n}=a_{n}\right\}=p_{a_{m}} \ldots p_{a_{n}},
$$

for all $m \leq n$ and $a_{m}, \ldots, a_{n} \in\{1, \ldots, k\}$. Denote by $\xi$ the partition into the following cylinders:

$$
\xi=\left\{\Delta_{1}, \ldots, \Delta_{k}\right\}, \quad \Delta_{n}=\left\{\omega=\left(\omega_{i}\right): \omega_{0}=n\right\} .
$$


It is not very difficult to see, that for $q \neq 1$

$$
h(\sigma, \nu, q, \xi)=-\frac{1}{q-1} \log \left(\sum_{i=1}^{k} p_{i}^{q}\right) .
$$

In particular, if $p_{1}=\ldots=p_{k}=1 / k$, then $h(\sigma, \nu, q, \xi)=\log k$. In this case, since $h(\sigma, \nu)=\log k$, we immediately conclude that $h(\sigma, \nu, q)=\log k$ for $q>1$.

We would also need the following statement.

Lemma 4.4. Suppose $\bar{T}: Y \rightarrow Y$ is an automorphism preserving a measure $\nu$. Then for any prime $p \geq 1$ one has

$$
h_{*}\left(\bar{T}^{p}, \nu\right)=p h_{*}(\bar{T}, \nu) .
$$

Proof. Assume first, that $(\bar{T}, \nu)$ is ergodic. If $\bar{T}^{p}: Y \rightarrow Y$ is ergodic, then there is nothing to prove, since in this case $h_{*}\left(\bar{T}^{p}, \nu\right)=h\left(\bar{T}^{p}, \nu\right)=p h(\bar{T}, \nu)$.

If $\bar{T}^{p}$ is not ergodic, then [17, p.38] there exist disjoint sets $A_{0}, \ldots, A_{p-1}$, such that $Y=\cup_{i=0}^{p-1} A_{i}(\bmod 0), \bar{T}\left(A_{i}\right)=A_{i+1} \bmod p$, and $\bar{T}^{p}$ is ergodic on $A_{0}$ with respect to $\nu\left(\cdot \mid A_{0}\right)$. Therefore, $h_{*}\left(\bar{T}^{p}, \nu\right)=p h(\bar{T}, \nu)$.

Hence, we conclude that if $(\bar{T}, \nu)$ is ergodic, then $h_{*}\left(\bar{T}^{p}, \nu\right)=p h(\bar{T}, \nu)$ for any prime $p, p \geq 1$.

Assume now that $(T, \mu)$ is not ergodic and let $\mu=\int \mu_{t} d m$ be the decomposition of $\mu$ into ergodic components. Applying the argument above to each $\left(T, \mu_{t}\right)$ we conclude that $h_{*}\left(T^{p}, \mu_{t}\right)=p h\left(T, \mu_{t}\right)$, and therefore $h_{*}\left(T^{p}, \mu\right)=p h_{*}(T, \mu)$.

Now let us proceed with the proof of the inequality: $h(T, \mu, q) \geq h_{*}(T, \mu)$ for all $q>1$. Assume the opposite, i.e., there exists $q>1$ such that $h(T, \mu, q)<h_{*}(T, \mu)$. Take a sufficiently large prime $p$ such that there exists an integer $k$ satisfying

$$
p h(T, \mu, q)<\log k<p h_{*}(T, \mu)=h_{*}\left(T^{p}, \mu\right) .
$$

Consider a Bernoulli shift, defined as above, with $p_{1}=\ldots=p_{k}=1 / k$. Then by Theorem 4.3 there exists a Bernoulli factor $Q$ for $T^{p}$ with $\mu\left(Q_{1}\right)=\ldots=\mu\left(Q_{k}\right)=1 / k$. Thus $h\left(T^{p}, \mu, q, Q\right)=\log k$, but this is in contradiction with (4.3), since

$$
p h(T, \mu, q)=h\left(T^{p}, \mu, q\right)=\sup _{R} h\left(T^{p}, \mu, q, R\right)
$$

- Hence, $h(T, \mu, q) \geq h_{*}(T, \mu)$ for $q>1$, and together with (4.2), this gives the equality $h(T, \mu, q)=h_{*}(T, \mu)$ for all $q>1$.

\section{RÉNyi ENTROPIES OF ORDER $q<1$}

In this section we will prove the remaining part of Theorem 1.2. The techniques which we are going to use will be different from the previous section. The reason is that we do not want to assume $h_{*}(T, \mu)>0$ (or, even $h(T, \mu)>0$ ). In the case when $h_{*}(T, \mu)>0$, we can (with the help of the non-ergodic version of Sinai's theorem on Bernoulli factors obtained in the previous section) proceed as in [22].

Our main goal is to construct partitions with arbitrarily large Rényi entropy of order $q, q<1$ : for every $C>0$ we have to find a partition $\xi$ such that

$$
h(T, \mu, q, \xi)=\liminf _{k \rightarrow \infty} \frac{1}{k} H_{\mu}\left(q, \xi^{(k)}\right) \geq C .
$$

Since the Rényi entropies are monotonic in $q$ we can restrict ourselves to $q \in(0,1)$. 
First of all, let us make an observation which will allow us to simplify the estimate of the Rényi entropy of a partition from below.

Definition 5.1. The Rényi entropy of order $q, q \neq 1$, of a finite partition $\eta=\left\{\Delta_{i}\right\}$ restricted to a set $F, \mu(F)>0$, is the number

$$
H_{\mu}(q, \eta \mid F)=-\frac{1}{q-1} \log \left(\sum_{\Delta_{i} \in \eta} \mu\left(\Delta_{i} \cap F\right)^{q}\right) .
$$

It is easy to see that any each $q \in(0,1)$ and for any set $F, \mu(F)>0$, one has

$$
H_{\mu}(q, \eta) \geq H_{\mu}(q, \eta \mid F) \text {. }
$$

In the next subsection we will show how this can be used when $F$ is a base of some Rokhlin-Halmos tower and $\xi$ is some special partition.

5.1. Rokhlin-Halmos towers and independent collections of sets. We have assumed that $T$ is an aperiodic automorphism. It is well known that for such automorphisms one can construct Rokhlin-Halmos towers of any height and measure arbitrarily close to 1.

Let $M \subset X$ then $\tau=\left\{M, T M, \ldots, T^{m-1} M\right\}$ is called a Rokhlin-Halmos tower if

$$
T^{i} M \cap T^{j} M=\varnothing \text { for } 0 \leq i \neq j \leq m-1 .
$$

We will use the same letter $\tau$ for $\cup_{k=0}^{m-1} T^{k} M$. The height of the tower $\tau$ is said to be $m$ and $\mu(\tau)=m \mu(M)$ is its measure.

We now give a definition of an independent collection of sets relative to a RokhlinHalmos tower. We will associate to such collections certain partitions, which will be analogous to Bernoulli partitions.

Definition 5.2. Let $\tau=\left\{M, T M, \ldots, T^{m-1} M\right\}$ be a Rokhlin-Halmos tower. We say that a collection $\mathcal{I}=\left\{I_{1}, \ldots, I_{N-1}\right\}$ of subsets of $\tau$ is independent relative to $\tau$ if

1) $I_{i} \cap I_{j}=\varnothing$ for $i \neq j$;

2) denote by $\xi_{\mathcal{I}}$ the partition of $X$ into the sets $\left\{I_{1}, \ldots, I_{N-1}, I_{N}:=X \backslash \cup_{j=0}^{N-1} I_{j}\right\}$, then

$$
\left\{T^{-k}\left(I_{1} \cap T^{k} M\right), \ldots, T^{-k}\left(I_{N} \cap T^{k} M\right)\right\}_{k=0}^{m-1}=\left\{T^{-k}\left(\xi_{\mathcal{I}} \cap T^{k} M\right)\right\}_{k=0}^{m-1}
$$

is a collection of independent partitions of $M$.

For convenience we will always assume that

3) $\mu\left(I_{j} \cap T^{k} M\right)=\frac{\mu(M)}{N}$ for $j=1, \ldots, N$ and $k=0, \ldots, m-1$.

Collections of independent sets exist in every tower. This follows from the following two observations. Firstly, since $T$ is assumed to be aperiodic, the invariant measure $\mu$ has no atoms. Secondly, for any Lebesgue space $(X, \mathfrak{B}, \mu)$, where $\mu$ has no atoms, for every measurable set $A$ and each $\alpha \in[0, \mu(A)]$ one can find a set $B \subseteq A$ with $\mu(B)=\alpha$.

It follows immediately from the definition 5.2 , that if $\mathcal{I}$ is a collection of independent sets in $\tau$ and $\xi_{\mathcal{I}}$ is the corresponding partition, then

$$
\xi_{\mathcal{I}}^{(k)} \cap M=\left\{I_{j_{1}} \cap T^{-1} I_{j_{2}} \cap \ldots T^{-k+1} I_{j_{k}} \cap M:\left(j_{1}, \ldots, j_{k}\right) \in\{1, \ldots, N\}^{k}\right\}
$$



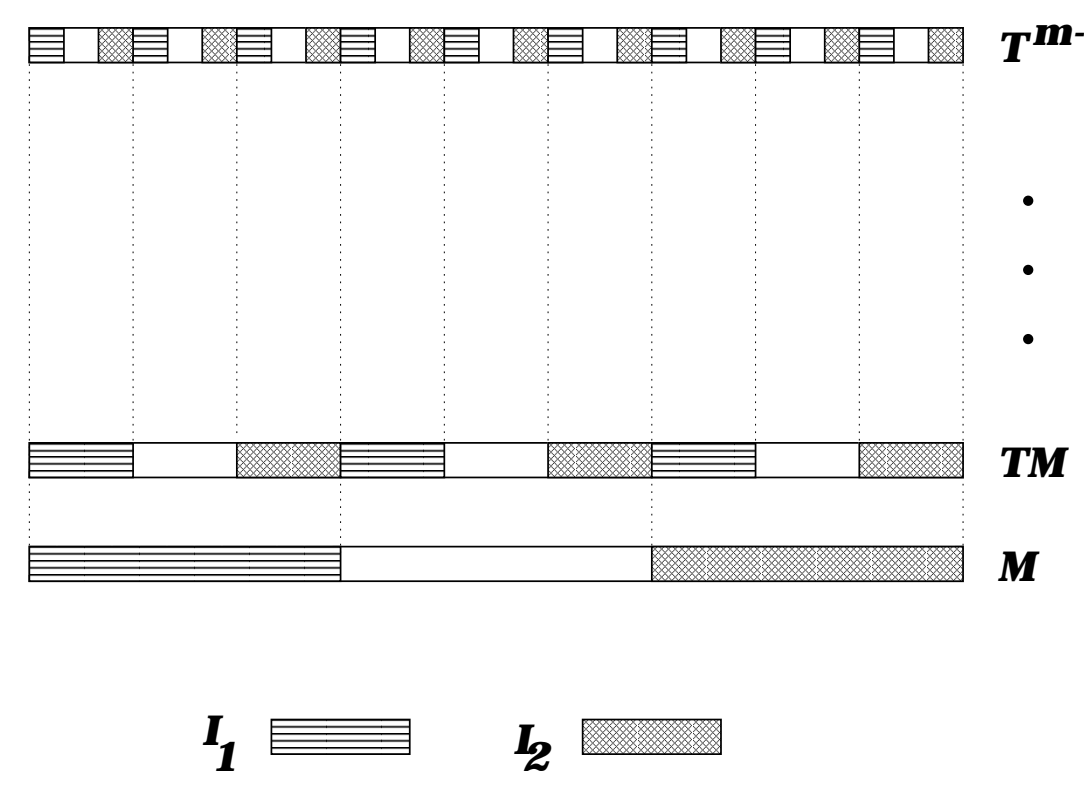

Figure 1. Independent sets $\left(I_{1}, I_{2}\right)$ in $\tau=\left(M, T M, \ldots, T^{m-1} M\right)$.

is a partition of $M$ into $N^{k}$ sets of equal measure $\mu(M) / N^{k}$ for every $k=1, \ldots, m$. Using (5.2) we easily obtain an estimate on the Rényi entropy of $\xi_{\mathcal{I}}^{(m)}$ :

$$
\begin{aligned}
H_{\mu}\left(q, \xi_{\mathcal{I}}^{(m)}\right) \geq H_{\mu}\left(q, \xi_{\mathcal{I}}^{(m)} \mid M\right) & =\frac{1}{1-q} \log N^{m}\left(\frac{\mu(M)}{N^{m}}\right)^{q} \\
& =m \log N+\frac{q}{1-q} \log \mu(M) .
\end{aligned}
$$

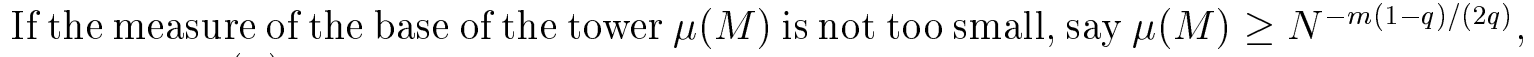
then $H_{\mu}\left(q, \xi_{\mathcal{I}}^{(m)}\right) / m \geq(\log N) / 2$.

In the next subsection we estimate the Rényi entropy of a partition which is 'close' to some partition $\xi_{\mathcal{I}}$, where $\mathcal{I}$ is a collection of independent sets.

5.2. Approximation lemma. Let $\mathcal{I}=\left(I_{1}, \ldots, I_{N-1}\right)$ be a collection of independent sets in the Rokhlin-Halmos $\tau=\left(M, \ldots, T^{m-1} M\right)$, and let $\xi_{\mathcal{I}}=\left\{I_{1}, \ldots, I_{N}\right\}$ be the corresponding partition. Suppose that another partition $\eta=\left(E_{1}, \ldots, E_{N}\right)$ is such that the sets

$$
E_{j}^{\tau}=E_{j} \cap \tau
$$

are "close" to the corresponding $I_{j}$ 's for $j=1, \ldots, N_{1}$. Since the partition $\xi_{\mathcal{I}}^{(m)}$ has a large Rényi entropy (subject to a relation between $N$ and $\mu(\tau)$ of course), then the partition $\eta^{(m)}$ has a large Rényi entropy as well. This can be rigorously formulated in the following way.

Lemma 5.3. Let $\mathcal{I}=\left\{I_{1}, \ldots, I_{N-1}\right\}$ be a collection of independent sets in $\tau=$ $\left(M, \ldots, T^{m-1} M\right)$ with $m \geq 16$, and let $\xi_{\mathcal{I}}=\left\{I_{1}, \ldots, I_{N-1}, I_{N}:=X \backslash \cup_{j=1}^{N-1} I_{j}\right\}$ be the 
corresponding partition. Suppose $\eta=\left(E_{1}, \ldots, E_{N-1}, E_{N}\right)$ is another partition of $X$ such that

$$
\sum_{j=1}^{N-1} \mu\left(E_{j}^{\tau} \triangle I_{j}\right) \leq \frac{\mu(\tau)}{16(N+31)} .
$$

Then for every $q \in(0,1)$ one has

$$
\frac{1}{m} H_{\mu}\left(q, \eta^{(m)}\right) \geq \frac{1}{4} \log N-\log 2+\frac{q}{(1-q) m} \log \mu(\tau)-\frac{q(1+q)}{2(1-q) m} \log (2 m) .
$$

Proof. This lemma is a generalization of lemmas 2.6 and 2.7 from [3], and its proof follows quite closely the proofs of the corresponding results in [3]. Nevertheless, due to the necessary modifications and for the sake of completeness we provide a proof here.

We shall use the following notation: let $\Omega=\{1, \ldots, N\}$ and

$$
\begin{aligned}
& \Delta(\mathbf{r})=I_{r_{1}} \cap T^{-1} I_{r_{2}} \cap \ldots \cap T^{-m+1} I_{r_{m}} \text { for } \mathbf{r}=\left(r_{1}, \ldots, r_{m}\right) \in \Omega^{m}, \\
& \tilde{\Delta}(\mathbf{s})=E_{s_{1}} \cap T^{-1} E_{s_{2}} \cap \ldots \cap T^{-m+1} E_{s_{m}} \text { for } \mathbf{s}=\left(s_{1}, \ldots, s_{m}\right) \in \Omega^{m} .
\end{aligned}
$$

We know that $\mu(\Delta(\mathbf{r}) \cap M)=\mu(M) / N^{m}$. Since $\eta$ is close to $\xi_{\mathcal{I}}$ in $\tau$, we expect the sets $\eta^{(m)} \cap M$ to have approximately the same measure as the sets of $\xi^{(m)} \cap M$. Let us make it precise. We say that $\tilde{\Delta}(\mathbf{s}), \mathbf{s} \in \Omega^{m}$, is a 'bad' (or, a 'fat') element of $\eta^{(m)}$ if

$$
\mu(\tilde{\Delta}(\mathbf{s}) \cap M) \geq 2^{m} N^{-m / 4} \mu(M),
$$

and is 'good' (or 'thin') otherwise. We collect the indexes of all 'bad' elements into the set

$$
S=\left\{\mathbf{s} \in \Omega^{m}: \tilde{\Delta}(\mathbf{s}) \text { is 'bad' }\right\} .
$$

We will now show that 'bad' elements of $\eta^{(m)}$ cover less than a half of $M$ in measure, i.e.,

$$
\mu\left(\bigcup_{\mathbf{s} \in S} \tilde{\Delta}(\mathbf{s}) \cap M\right) \leq \frac{1}{2} \mu(M) .
$$

We introduce the following notation:

$$
\begin{aligned}
M(\mathbf{s}, \mathbf{r}) & =\tilde{\Delta}(\mathbf{s}) \cap \Delta(\mathbf{r}) \cap M, \\
\tau(\mathbf{s}, \mathbf{r}) & =\bigcup_{k=0}^{m-1} T^{k} M(\mathbf{s}, \mathbf{r}), \\
\tau(\mathbf{s}) & =\bigcup_{\mathbf{r} \in \Omega^{m}} \tau(\mathbf{s}, \mathbf{r}) .
\end{aligned}
$$

It is easy to see that,

$$
\begin{gathered}
M(\mathbf{s}, \mathbf{r}) \cap M(\mathbf{s}, \mathbf{t})=\varnothing \quad \text { for } \quad \mathbf{r} \neq \mathbf{t}, \\
T^{i} M(\mathbf{s}, \mathbf{r}) \cap T^{j} M(\mathbf{s}, \mathbf{r})=\varnothing \quad \text { for } \quad i \neq j, \\
\tau(\mathbf{s}, \mathbf{r}) \cap \tau(\mathbf{s}, \mathbf{t})=\varnothing \quad \text { for } \quad \mathbf{r} \neq \mathbf{t} .
\end{gathered}
$$


Let $\mathbf{s} \in \Omega^{m}$, then

$$
\begin{aligned}
\mu\left(\left(\cup_{j=1}^{N-1} E_{j}^{\tau} \triangle I_{j}\right) \cap \tau(\mathbf{s})\right) & =\sum_{\mathbf{r} \in \Omega^{m}} \mu\left(\left(\cup_{j=1}^{N-1} E_{j}^{\tau} \triangle I_{j}\right) \cap \tau(\mathbf{s}, \mathbf{r})\right) \\
& =\sum_{\mathbf{r} \in \Omega^{m}} \sum_{k=0}^{m-1} \mu\left(\left(\cup_{j=1}^{N-1} E_{j}^{\tau} \triangle I_{j}\right) \cap T^{k} M(\mathbf{s}, \mathbf{r})\right)
\end{aligned}
$$

Consider the sets participating in the last sum separately. We claim that

$$
\left(\cup_{j=1}^{N-1} E_{j}^{\tau} \triangle I_{j}\right) \cap T^{k} M(\mathbf{s}, \mathbf{r})= \begin{cases}T^{k} M(\mathbf{s}, \mathbf{r}), & \text { if } s_{k} \neq r_{k} \\ \varnothing, & \text { if } s_{k}=r_{k} .\end{cases}
$$

For this it is sufficient to show that

$$
\left(\cup_{j=1}^{N-1} E_{j}^{\tau} \triangle I_{j}\right) \cap E_{s_{k}}^{\tau} \cap I_{r_{k}}= \begin{cases}E_{s_{k}}^{\tau} \cap I_{r_{k}} & \text { if } s_{k} \neq r_{k}, \\ \varnothing, & \text { if } s_{k}=r_{k} .\end{cases}
$$

The proof is straightforward: let $j=1, \ldots, N-1$ and $k=1, \ldots, m$, then

$$
\begin{aligned}
\left(E_{j}^{\tau} \triangle I_{j}\right) \cap E_{s_{k}}^{\tau} \cap I_{r_{k}} & =\left(\left(E_{j}^{\tau} \backslash I_{j}\right) \cap E_{s_{k}}^{\tau} \cap I_{r_{k}}\right) \cup\left(\left(I_{j} \backslash E_{j}^{\tau}\right) \cap E_{s_{k}}^{\tau} \cap I_{r_{k}}\right) \\
= & : A \cup B .
\end{aligned}
$$

Suppose first that $s_{k}=r_{k}$. Then for $j=s_{k}=r_{k}$ we have

$$
A \cup B \subseteq\left(\left(E_{j}^{\tau} \backslash I_{j}\right) \cap I_{j}\right) \cup\left(\left(I_{j} \backslash E_{j}^{\tau}\right) \cap E_{j}\right)=\varnothing
$$

since $I_{j} \subset \tau$ for $j=1, \ldots, N-1$.

For $j \neq s_{k}=r_{k}$ we have

$$
A \cup B \subseteq\left(\left(E_{j}^{\tau} \backslash I_{j}\right) \cap E_{s_{k}}^{\tau}\right) \cup\left(\left(I_{j} \backslash E_{j}^{\tau}\right) \cap I_{r_{k}}\right)=\varnothing,
$$

since $E_{j} \cap E_{s_{k}}=I_{j} \cap I_{r_{k}}=\varnothing$.

Now consider the case $s_{k} \neq r_{k}$. If $j \neq s_{k}$ and $j \neq r_{k}$, then

$$
A \cup B \subseteq\left(E_{j} \cap E_{s_{k}}\right) \cup\left(I_{j} \cap I_{r_{k}}\right)=\varnothing .
$$

If $j \neq s_{k}$ and $j=r_{k}$, then $A \subseteq\left(E_{j}^{\tau} \backslash I_{j}\right) \cap E_{s_{k}}=\varnothing$, but

$$
B=\left(I_{j} \backslash E_{j}^{\tau}\right) \cap E_{s_{k}} \cap I_{r_{k}}=\left(I_{r_{k}} \cap E_{s_{k}}^{\tau}\right),
$$

since $E_{j}^{\tau} \cap E_{s_{k}}^{\tau}=\varnothing$.

Similarly, for $j=s_{k}$ and $j \neq r_{k}$, we conclude that $B=\varnothing$, but $A=E_{s_{k}}^{\tau} \cap I_{r_{k}}$. Hence we proved (5.6), and therefore (5.5).

Using (5.5) and the fact that $T$ is measure-preserving, we can simplify (5.4):

$$
\mu\left(\left(\cup_{j=1}^{N-1} E_{j}^{\tau} \triangle I_{j}\right) \cap \tau(\mathbf{s})\right)=\sum_{\mathbf{r} \in \Omega^{m}} d_{H}(\mathbf{s}, \mathbf{r}) \mu(M(\mathbf{s}, \mathbf{r})),
$$

where $d_{H}(\mathbf{s}, \mathbf{r})=\#\left\{k: s_{k} \neq r_{k}\right\}$ is the Hamming distance between $\mathbf{s}$ and $\mathbf{r}$. We rewrite (5.7) in the following form

$$
\mu\left(\left(\cup_{j=1}^{N-1} E_{j}^{\tau} \triangle I_{j}\right) \cap \tau(\mathbf{s})\right)=\sum_{i=0}^{m} i \sum_{\mathbf{r}: d_{H}(\mathbf{s}, \mathbf{r})=i} \mu(M(\mathbf{s}, \mathbf{r})) .
$$


Given $\mathbf{s} \in \Omega^{m}$ the number of $\mathbf{r}$ 's such that $d_{H}(\mathbf{s}, \mathbf{r})=i$ is $C_{m}^{i}(N-1)^{i}$, where $C_{m}^{i}$ is the binomial coefficient. Let us introduce the following notation

$$
x_{i}(\mathbf{s}):=\sum_{\mathbf{r}: d_{H}(\mathbf{s}, \mathbf{r})=i} \mu(M(\mathbf{s}, \mathbf{r})), \quad y_{i}:=\frac{\mu(M)}{N^{m}} C_{m}^{i}(N-1)^{i} .
$$

Note that $\mu(\tilde{\Delta}(\mathbf{s}) \cap M)=\sum_{i=0}^{m} x_{i}(\mathbf{s})$.

Since $M(\mathbf{s}, \mathbf{r}) \subset \Delta(\mathbf{r}) \cap M$ and $\mu(\Delta(\mathbf{r}) \cap M)=\mu(M) / N^{m}$, for every $i$ one has

$$
x_{i}(\mathbf{s})=\sum_{\mathbf{r}: d_{H}(\mathbf{s}, \mathbf{r})=i} \mu(M(\mathbf{s}, \mathbf{r})) \leq \frac{\mu(M)}{N^{m}}\left[\sum_{\mathbf{r}: d_{H}(\mathbf{s}, \mathbf{r})=i} 1\right]=\frac{\mu(M)}{N^{m}} C_{m}^{i}(N-1)^{i}=y_{i} .
$$

Furthermore, for every s there exists $k_{\mathbf{s}} \in\{1, \ldots, m\}$ such that

$$
\sum_{i=0}^{k_{\mathbf{s}}} y_{i}>\sum_{i=0}^{m} x_{i}(\mathbf{s}) \geq \sum_{i=0}^{k_{\mathbf{s}}-1} y_{i}
$$

From (5.9) and (5.10) we conclude that $\sum_{i=l}^{m} x_{i}(\mathbf{s}) \geq \sum_{i=l}^{k_{\mathbf{s}}-1} y_{i}$ for all $l \geq 0$, and as a result

$$
\sum_{i=0}^{m} i x_{i}(\mathbf{s}) \geq \sum_{i=0}^{k_{\mathbf{s}}-1} i y_{i}
$$

We will show now that if $\mathbf{s} \in S$ then $k_{\mathbf{s}} \geq\left[\frac{3 m}{4}\right]+1$. Indeed, if $\mathbf{s} \in S$, then by definition of $S$,

$$
\mu(\tilde{\Delta}(\mathbf{s}) \cap M) \geq 2^{m} N^{-m / 4} \mu(M),
$$

and from (5.9) we have

$$
\frac{1}{N^{m}} \sum_{i=0}^{k_{s}} C_{m}^{i}(N-1)^{i} \geq \frac{\sum_{i=0}^{m} x_{i}(\mathbf{s})}{\mu(M)}=\frac{\mu(\tilde{\Delta}(\mathbf{s}) \cap M)}{\mu(M)} \geq 2^{m} N^{-m / 4} .
$$

However, Lemma 6.1 (see Appendix below) states that

$$
\frac{1}{N^{m}} \sum_{i=0}^{k} C_{m}^{i}(N-1)^{i}<2^{m} N^{-m / 4}
$$

for all $k=0,1, \ldots,[3 m / 4]$. Hence, $k_{\mathbf{s}} \geq[3 m / 4]+1$. 
Now, for all $\mathrm{s} \in S$ we have

$$
\begin{aligned}
& \mu\left(\left(\cup_{j=1}^{N-1} E_{j}^{\tau} \triangle I_{j}\right) \cap \tau(\mathbf{s})\right)=\sum_{i=0}^{m} i x_{i}(\mathbf{s}) \quad(\text { by }(5.8)) \\
& \geq \sum_{i=0}^{k_{\mathbf{s}}-1} i y_{i}=\frac{\mu(M)}{N^{m}} \sum_{i=0}^{k_{\mathbf{s}}-1} i C_{m}^{i}(N-1)^{i} \quad(\text { by }(5.11)) \\
& \geq \frac{\mu(M)}{N^{m}} \frac{m}{8(N+31)} \sum_{i=0}^{k_{\mathrm{s}}} C_{m}^{i}(N-1)^{i} \quad \text { (by Lemma 6.1) } \\
& =\frac{m}{8(N+31)} \sum_{i=0}^{k_{\mathbf{s}}} y_{i} \\
& \left.\geq \frac{m}{8(N+31)} \sum_{i=0}^{m} x_{i}(\mathbf{s}) \quad \text { (by }(5.10)\right) \text {. }
\end{aligned}
$$

Hence,

$$
\begin{aligned}
\mu & \left(\cup_{j=1}^{N-1} E_{j}^{\tau} \Delta I_{j}\right) \geq \sum_{\mathbf{s} \in S} \mu\left(\left(\cup_{j=1}^{N-1} E_{j}^{\tau} \Delta I_{j}\right) \cap \tau(\mathbf{s})\right) \\
& \geq \frac{m}{8(N+31)} \sum_{\mathbf{s} \in S} \sum_{i=0}^{m} x_{i}(\mathbf{s})=\frac{m}{8(N+31)} \sum_{\mathbf{s} \in S} \sum_{\mathbf{r} \in \Omega^{m}} \mu(M(\mathbf{s}, \mathbf{r})) \\
& =\frac{m}{8(N+31)} \sum_{\mathbf{s} \in S} \mu(\tilde{\Delta}(\mathbf{s}) \cap M) .
\end{aligned}
$$

Therefore, using our assumption (5.3) one has

$$
\begin{aligned}
\sum_{\mathbf{s} \in S} \mu(\tilde{\Delta}(\mathbf{s}) \cap M) & \leq \frac{8(N+31)}{m} \mu\left(\cup_{j=1}^{N-1} E_{j}^{\tau} \triangle I_{j}\right) \\
& \leq \frac{8(N+31)}{16(N+31) m} \mu(\tau)=\frac{1}{2} \mu(M),
\end{aligned}
$$

i.e., 'bad' elements $\tilde{\Delta}(\mathrm{s})$ cover not more than a half of $M$. 
Now we can estimate the Rényi entropy of the partition $\eta^{(m)}$ :

$$
\begin{aligned}
H_{\mu}\left(q, \eta^{(m)}\right) & \geq H_{\mu}\left(q, \eta^{(m)} \mid M\right)=\frac{1}{1-q} \log \left(\sum_{\mathbf{s} \in \Omega^{m}} \mu(\tilde{\Delta}(\mathbf{s}) \cap M)^{q}\right) \\
& \geq \frac{1}{1-q} \log \left(\sum_{\mathbf{s} \in \Omega^{m} \backslash S} \mu(\tilde{\Delta}(\mathbf{s}) \cap M)^{q}\right) \\
& \geq \frac{1}{1-q} \log \left(\sum_{\mathbf{s} \in \Omega^{m} \backslash S} \frac{\mu(\tilde{\Delta}(\mathbf{s}) \cap M)}{\left(2^{m} N^{-m / 4} \mu(M)\right)^{1-q}}\right) \\
& \geq \frac{1}{1-q} \log \left(\frac{1}{2} \frac{\mu(M)}{\left(2^{m} N^{-m / 4} \mu(M)\right)^{1-q}}\right) \\
& =\frac{m}{4} \log N-m \log 2+\frac{q}{1-q} \log \mu(M)-\frac{1}{1-q} \log 2 \\
& =m\left(\frac{1}{4} \log N-\log 2+\frac{q}{(1-q) m} \log \mu(\tau)\right)-\frac{1}{1-q} \log 2 m^{q} .
\end{aligned}
$$

This finishes the proof of Lemma 5.3.

5.3. Partitions with large Rényi entropy. Consider $q \in(0,1)$ and take $N \in \mathbb{N}$, $N \geq 16$. For the convenience of notation we put $\delta=\frac{(1-q)}{8 q}$. Take $R \in \mathbb{N}$ such that

$$
N^{\delta R}>32(N+31) N
$$

We choose a sequence of Rokhlin-Halmos towers $\left\{\tau_{k}\right\}$,

$$
\tau_{k}=\left(M_{k}, \ldots, T^{m_{k}-1} M_{k}\right)
$$

of height $m_{k}=R k$ and measure $\mu\left(\tau_{k}\right)=N^{-\delta R k}$. For each $k$ let

$$
\mathcal{I}_{k}=\left(I_{1}(k), \ldots, I_{N-1}(k)\right)
$$

be a collection of independent sets in $\tau_{k}$. We define a sequence of collections of pairwise disjoint sets $\mathcal{E}_{k}=\left(E_{1}(k), \ldots, E_{N-1}(k)\right)$ as follows, for $j=1, \ldots, N-1$ let

$$
\begin{aligned}
& E_{j}(0)=\varnothing, \\
& E_{j}(k)=\left(E_{j}(k-1) \backslash \tau_{k}\right) \cup I_{j}(k) \quad \text { for } k=1,2, \ldots .
\end{aligned}
$$

For any $j \in\{1, \ldots, N-1\}$ the sequence of characteristic functions $\left\{\chi_{E_{j}(k)}\right\}_{k=1}^{\infty}$ is a Cauchy sequence in $L_{1}(X, \mathfrak{B}, \mu)$. Indeed, we obviously have

$$
E_{j}(k) \triangle E_{j}(k-1) \subseteq \tau_{k},
$$

and hence for $k_{1}, k_{2} \geq K$ we have

$$
\mu\left(E_{j}\left(k_{1}\right) \triangle E_{j}\left(k_{2}\right)\right) \leq \sum_{k=K}^{+\infty} \mu\left(\tau_{k}\right) \rightarrow 0 \text { as } K \rightarrow \infty .
$$

From this we conclude that there exists $E_{j} \in \mathfrak{B}$ such that

$$
\chi_{E_{j}(k)} \rightarrow \chi_{E_{j}} \text { for } k \rightarrow \infty \text {. }
$$


It follows from the construction that $\mu\left(E_{j} \cap E_{i}\right)=0$ for $i \neq j$. Since we can neglect sets of measure zero we may assume that $E_{i} \cap E_{j}=\varnothing$ and hence we have a collection $\mathcal{E}=\left(E_{1}, \ldots, E_{N-1}\right)$ of pairwise disjoint subsets of $X$.

Furthermore, for every $j=1, \ldots, N-1$ and any $L>k$ one has

$$
\begin{gathered}
\mu\left(E_{j}^{\tau_{k}} \Delta I_{j}(k)\right) \leq \mu\left(E_{j}^{\tau_{k}} \Delta E_{j}^{\tau_{k}}(L)\right)+\sum_{l=k}^{L-1} \mu\left(E_{j}^{\tau_{k}}(l+1) \Delta E_{j}^{\tau_{k}}(l)\right) \\
+\mu\left(E_{j}^{\tau_{k}}(k) \triangle I_{j}(k)\right) .
\end{gathered}
$$

Moreover, since $E_{j}^{\tau_{k}}(k)=I_{j}(k), \mu\left(E_{j} \triangle E_{j}(L)\right) \rightarrow 0$ as $L \rightarrow \infty$, and $E_{j}(l+1) \Delta$ $E_{j}(l) \subseteq \tau_{l}$, we conclude that

$$
\mu\left(E_{j}^{\tau_{k}} \triangle I_{j}(k)\right) \leq \sum_{l=k+1}^{\infty} \mu\left(\tau_{l}\right)=\mu\left(\tau_{k}\right) \frac{N^{-\delta R}}{1-N^{-\delta R}}<\frac{\mu\left(\tau_{k}\right)}{16(N+31) N},
$$

and hence

$$
\sum_{j=1}^{N-1} \mu\left(E_{j}^{\tau_{k}} \Delta I_{j}(k)\right)<\frac{\mu\left(\tau_{k}\right)}{16(N+31)} .
$$

Now let $\eta=\left\{E_{1}, \ldots, E_{N-1}, E_{N}\right\}$, where $E_{N}=X \backslash \cup_{j=1}^{N-1} E_{j}$, and applying Lemma 5.3 we conclude that

$$
\begin{aligned}
\frac{1}{m_{k}} H_{\mu}\left(q, \eta^{\left(m_{k}\right)}\right) & \geq \frac{1}{4} \log N+\frac{q}{(1-q) m_{k}} \log \mu\left(\tau_{k}\right)+o(1) \\
& =\frac{1}{8} \log N+o(1),
\end{aligned}
$$

where $o(1) \rightarrow 0$ as $k \rightarrow \infty$.

For any $n \in \mathbb{N}$ there exists $k \in \mathbb{N}$ such that

$$
m_{k}=R k \leq n<R(k+1)=m_{k+1} .
$$

Since $H_{\mu}\left(q, \eta^{(n)}\right) \geq H_{\mu}\left(q, \eta^{\left(m_{k}\right)}\right)$, we have

$$
\frac{1}{n} H_{\mu}\left(q, \eta^{(n)}\right) \geq \frac{m_{k}}{m_{k+1}} \frac{1}{m_{k}} H_{\mu}\left(q, \eta^{\left(m_{k}\right)}\right) \geq \frac{1}{1+\frac{1}{k}}\left(\frac{1}{8} \log N+o(1)\right) .
$$

This proves that

$$
h_{\mu}(T, q, \eta)=\liminf _{n \rightarrow \infty} \frac{1}{n} H_{\mu}\left(q, \eta^{(n)}\right) \geq \frac{1}{9} \log N .
$$

Everywhere above we have assumed that $q \in(0,1)$. However, since

$$
h(T, \mu, q, \xi) \geq h(T, \mu, 1 / 2, \xi)
$$

for all $q \leq 0$ and every partition $\xi$, we have obtained partitions with large Rényi entropies of all orders $q, q \leq 0$, as well. Finally, since $N$ is an arbitrary integer, we proved the remaining part of Theorem 1.2. 
a) Another version of Rényi entropies can be defined using lim sup instead of lim inf in (2.2). In principle, due to the lack of subadditivity of $H_{q}$, there might exist a finite partition $\xi$ such that

$$
\begin{aligned}
\bar{h}(T, \mu, q, \xi):=\limsup _{n \rightarrow \infty} & -\frac{1}{(q-1) n} H_{\mu}\left(q, \xi^{(n)}\right) \neq \\
& \liminf _{n \rightarrow \infty}-\frac{1}{(q-1) n} H_{\mu}\left(q, \xi^{(n)}\right)=h(T, \mu, q, \xi)
\end{aligned}
$$

for some $q \in \mathbb{R}, q \neq 1$. However, using the results of Theorems 1.1 and 1.2 , one can easily show that

$$
\bar{h}(T, \mu, q):=\sup _{\xi \text { finite }} \bar{h}(T, \mu, q, \xi)=\sup _{\xi \text { finite }} h(T, \mu, q, \xi)=h(T, \mu, q)
$$

for all $q \in \mathbb{R}$.

Since $h(T, \mu, q)=+\infty$ for $q<1$ in the ergodic and aperiodic cases, the claim is obviously true for $q<1$.

To complete the proof we have to show that for $q>1$

$$
\sup _{\xi \text { finite }} \bar{h}(T, \mu, q, \xi) \leq h(T, \mu)
$$

in the ergodic case, and

$$
\sup _{\xi \text { finite }} \bar{h}(T, \mu, q, \xi) \leq h_{*}(T, \mu)
$$

in the aperiodic case. The first inequality (6.1) follows immediately from the monotonicity properties (Proposition 2.1), and the fact that for $q=1$ (standard entropy) the limit in (2.2) exists.

The second inequality (6.2) is proved exactly in the same manner as an inequality $h(T, \mu, q) \leq h_{*}(T, \mu)$ in section 4.1 .

b) Formally speaking, the pair of metric invariants $(h(T, \mu), h(T, \mu, q)), q>1$, can detect ergodicity: if $h(T, \mu)-h(T, \mu, q)>0$, then $(T, \mu)$ cannot be ergodic. However, we were not able to find any relevant examples where this could be useful.

In our opinion, an example of a measure-preserving system $(X, \mathfrak{B}, \mu, T)$, where the non-ergodicity can be decided from the positiveness of $h(T, \mu)-h(T, \mu, q)$ would be interesting.

c) The difference between ergodicity and non-ergodicity is less interesting than the difference between ergodicity and weak mixing. As it is well known, weak mixing of $T$ is equivalent to the ergodicity of any direct products of $T$ with an ergodic automorphism $S$. Suppose, $T$ is ergodic, but not weakly mixing. Then there exists an ergodic measure-preserving dynamical system $(Y, \mathfrak{F}, \nu, S)$ such that $(X \times Y, \mathfrak{G}, \mu \times$ $\nu, T \times S)$ is not ergodic. Unfortunately, the Rényi entropies are not able to detect non-ergodicity of such systems: for $q>1$ one has

$$
h(T \times S, \mu \times \nu, q)=h(T, \mu, q)+h(S, \nu, q)=h(T, \mu)+h(S, \nu)=h(T \times S, \mu \times \nu),
$$

where the first and the third equalities are standard facts for entropy-like characteristics, and the second equality follows from Theorem 1.1 
d) Entropy convergence rates were introduced in [3].

Let $(X, \mathfrak{B}, \mu)$ be a Lebesgue space and $T$ be a measure-preserving automorphism. Suppose that $(X, \mathfrak{B}, \mu, T)$ has zero entropy. Hence, for any finite partition $\xi$ one has

$$
h(T, \mu, \xi)=\liminf _{n \rightarrow \infty} \frac{1}{n} H_{\mu}\left(\xi^{(n)}\right)=0 .
$$

Let $c>0$ and $a_{n}, n \geq 1$, is a sequence of positive numbers such that $a_{n} \rightarrow \infty$. Denote by $\Pi$ the set of all non-trivial partitions $(X, \mathfrak{B}, \mu)$ into two sets.

The automorphism $T$ is said to be

- of type $(L I \geq c)$ for $\left(\left(a_{n}\right), \Pi\right)$ if for every $\xi \in \Pi$

$$
\liminf _{n \rightarrow \infty} \frac{1}{a_{n}} H\left(\xi^{(n)}\right) \geq c ;
$$

- of type $(L S \geq c)$ for $\left(\left(a_{n}\right), \Pi\right)$ if for every $\xi \in \Pi$

$$
\limsup _{n \rightarrow \infty} \frac{1}{a_{n}} H\left(\xi^{(n)}\right) \geq c .
$$

Similarly one defines types $(L I \leq c),(L S \leq c),(L I<c)$, etc. Clearly, the type of a measure-preserving transformation is a measure-theoretic invariant.

It was shown in [3] that there are no aperiodic transformations of type $(L I<\infty)$ for $\left(\left(a_{n}\right), \Pi\right)$, where $a_{n}=o(n), n \geq 1$. Every totally ergodic transformation (i.e., $T^{k}$ is ergodic for every $k \geq 1)$ is of type $(L S=\infty)$ for $(g(\log n)$, $\Pi)$, where $g:[0,+\infty) \rightarrow \mathbf{R}$ is positive, monotone increasing and

$$
\int_{1}^{\infty} \frac{g(x)}{x^{2}} d x<\infty
$$

Also, in [2] F.Blume constructed a class of weakly mixing systems, which can be distinguished by these invariants.

It would interesting to know if the corresponding notions for the Rényi entropies, both in the case of $q<1$ and $q>1$, can produce useful convergence rates, which are different from the case of standard entropy.

\section{Appendix. Auxiliary Results}

Throughout this section we assume that $N \in \mathbb{N}, N \geq 2,[x]$ denotes an integer part of $x$, and $C_{m}^{k}$ will denote the binomial coefficient

$$
C_{m}^{k}=\frac{m !}{k !(m-k) !}
$$

For $k=0, \ldots, m$ let

$$
a_{m}(k, N)=\sum_{i=0}^{k} C_{m}^{i}(N-1)^{i}, \quad b_{m}(k, N)=\sum_{i=0}^{k-1} i C_{m}^{i}(N-1)^{i} .
$$

The following result is a straightforward generalization of Lemma 2.4 from [3].

Lemma 6.1. Let $m$ be an integer, $m \geq 16$, and put $k_{0}=\left[\frac{3 m}{4}\right]+1$. Then 
1) for $k=0,1, \ldots, k_{0}-1$ one has

$$
\frac{a_{m}(k, N)}{N^{m}} \leq 2^{m} N^{-m / 4}
$$

2) for $k=k_{0}, \ldots, m$ one has

$$
\frac{a_{m}(k, N)}{b_{m}(k, N)} \leq \frac{8(N+31)}{m} .
$$

\section{ACKNOWLEDGMENTS}

We are grateful to F. Blume and E.G.F. Thomas for valuable disscusions. The second author acklowledges the support of the Dutch Organization for Scientific Research (NWO), grant 613-06-551.

\section{REFERENCES}

[1] C. Beck and F. Schlögl. Thermodynamics of chaotic systems. Cambridge University Press, Cambridge, 1993. An introduction.

[2] F. Blume. Fast entropy convergence for rank one systems. Technical report, 1997.

[3] F. Blume. Possible rates of entropy convergence. Ergodic Theory Dynam. Systems, 17(1):45-70, 1997.

[4] I. P. Cornfeld, S. V. Fomin, and Y. G. Sină. Ergodic theory. Springer-Verlag, New York, 1982. Translated from the Russian by A. B. Sosinskilu.

[5] M. Denker, C. Grillenberger, and K. Sigmund. Ergodic theory on compact spaces. SpringerVerlag, Berlin, 1976. Lecture Notes in Mathematics, Vol. 527.

[6] J.-P. Eckmann and D. Ruelle. Ergodic theory of chaos and strange attractors. Rev. Modern Phys., 57(3, part 1):617-656, 1985.

[7] P. Grassberger and I. Procaccia. Dimensions and entropies of strange attractors from a fluctuating dynamics approach. Phys. D, 13(1-2):34-54, 1984.

[8] H. G. E. Hentschel and I. Procaccia. The infinite number of generalized dimensions of fractals and strange attractors. Phys. D, 8(3):435-444, 1983.

[9] J. C. Kieffer. On the minimum number of fixed length sequences with fixed total probability. Ann. Probability, 4(2):335-337, 1976.

[10] J. C. Kieffer and M. Rahe. Selecting universal partitions in ergodic theory. Ann. Probab., 9(4):705-709, 1981.

[11] W. Krieger. On entropy and generators of measure-preserving transformations. Trans. Amer. Math. Soc., 149:453-464, 1970.

[12] D. S. Ornstein. Ergodic theory, randomness, and dynamical systems. Yale University Press, New Haven, Conn., 1974. James K. Whittemore Lectures in Mathematics given at Yale University, Yale Mathematical Monographs, No. 5.

[13] Y. B. Pesin. Dimension theory in dynamical systems. Chicago Lectures in Mathematics. University of Chicago Press, Chicago, IL, 1997. Contemporary views and applications.

[14] A. Rényi. Probability theory. North-Holland Publishing Co., Amsterdam, 1970. Translated by László Vekerdi, North-Holland Series in Applied Mathematics and Mechanics, Vol. 10.

[15] V. A. Rohlin. On the fundamental ideas of measure theory. Mat. Sbornik N.S., 25(67):107-150, 1949.

[16] V. A. Rokhlin. Izbrannye raboty. Moskovskii Tsentr Nepreryvnogo Matematicheskogo Obrazovaniya, Moscow, 1999. Reminiscences of V. A. Rokhlin by V. I. Arnol'd, A. M. Vershik, S. P. Novikov and Ya. G. Sinai, Edited and with a preface by Vershik.

[17] D. J. Rudolph. Fundamentals of measurable dynamics. The Clarendon Press Oxford University Press, New York, 1990. Ergodic theory on Lebesgue spaces.

[18] P. Shields. The theory of Bernoulli shifts. The University of Chicago Press, Chicago, Ill.-London, 1973. Chicago Lectures in Mathematics. 
[19] J. G. Sinaŭ. On a weak isomorphism of transformations with invariant measure. Mat. Sb. (N.S.), 63 (105):23-42, 1964.

[20] M. Smorodinsky. Ergodic theory, entropy. Springer-Verlag, Berlin, 1971. Lecture Notes in Mathematics, Vol. 214.

[21] ك̌. Šujan. Generators of an abelian group of invertible measure-preserving transformations. Monatsh. Math., 90(1):67-79, 1980.

[22] F. Takens and E. Verbitski. Generalized entropies: Rényi and correlation integral approach. Nonlinearity, 11(4):771-782, 1998.

[23] K. Winkelbauer. On the asymptotic rate of non-ergodic information sources. Kybernetika (Prague), 6:127-148, 1970.

DEPARTMENT OF MATHEMATICS, UNIVERSITY OF GRONINGEN, P.O.BOX 800, 9700 AV, GRONINGEN, THE NETHERLANDS

E-mail address: F.TAKENS@MATH.RUG.NL

EURANDOM, P.O.BOX 512, 5600 MB, EINDHOVEN, THE NETHERLANDS

E-mail address: E.VERBITSKIY@TUE.NL 